\title{
Characterization of Turmeric and Curry Samples by Liquid Chromatography with Spectroscopic Detection Based on Polyphenolic and Curcuminoid Contents
}

\author{
Oscar Vidal-Casanella ${ }^{1}$, Nerea Nuñez ${ }^{1}$, Sonia Sentellas ${ }^{1}$, Oscar Núñez ${ }^{1,2,3}$ \\ and Javier Saurina $1,2, *$ (D) \\ 1 Department of Chemical Engineering and Analytical Chemistry, University of Barcelona. Martí i Franquès, \\ 1-11, E-08028 Barcelona, Spain; oscarvidalcasanella@gmail.com (O.V.-C.); nereant7@gmail.com (N.N.); \\ ssentellas@hotmail.com (S.S.); oscar.nunez@ub.edu (O.N.) \\ 2 Research Institute in Food Nutrition and Food Safety, University of Barcelona, Recinte Torribera, Av. Prat de \\ la Riba 171, Edifici de Recerca (Gaudí), Santa Coloma de Gramenet, E-08921 Barcelona, Spain \\ 3 Serra Húnter Fellow, Generalitat de Catalunya, E08007 Barcelona, Spain \\ * Correspondence: xavi.saurina@ub.edu
}

Received: 19 March 2020; Accepted: 17 April 2020; Published: 21 April 2020

\begin{abstract}
This paper deals with the characterization of turmeric and related products using the compositional fingerprints of curcuminoids (e.g., curcumin, demethoxycurcumin and bisdemethoxycurcumin) and other phenolic compounds (e.g., hydroxybenzoic and hydroxycinnamic acids and flavonoids) as the source of analytical information. Under this approach, the quantitative determination of analytes becomes unnecessary and even data from unknown components can be advantageously exploited for sample exploration and authentication. The methodology relied on sample extraction with hydro-organic solvents to recover the components of interest and further analysis of the corresponding extracts by liquid chromatography with diode array detection (HPLC-DAD). Extraction conditions were optimized focusing on the independent recovery of curcuminoids and polyphenols. Two different HPLC methods under reversed-phase mode were used to generate the chromatographic fingerprints at 420 and $280 \mathrm{~nm}$ for the specific monitoring of curcuminoids and polyphenols, respectively. Both extraction and separation steps were optimized under experimental design approaches to achieve the richest compositional fingerprints in terms of variety of components. The resulting data was subsequently treated chemometrically by principal component analysis (PCA) and related classification methods to achieve a better overall description of samples. Polyphenolic fingerprints were appropriate to discriminate among turmeric and mixed spices, while curcuminoid fingerprints could be useful to distinguish turmeric varieties.
\end{abstract}

Keywords: liquid chromatography; experimental design; turmeric characterization; polyphenolic fingerprints; curcuminoid fingerprints; principal component analysis

\section{Introduction}

Turmeric has been used for centuries as a spice or condiment in the traditional cuisine of many Asian countries [1]. Apart from its characteristic complex flavor, it is also appreciated as a colorant providing a yellow hue to the dishes [2]. Besides, turmeric is rich in active components with antibacterial, anti-inflammatory, anti-diabetic and antioxidant activities so it has been used in traditional Asian medicine as well [3-6]. Among other components, phenolic compounds, curcumin and related species have been found to be responsible for those beneficial properties. Owing to the exceptional pharmaceutical features of curcuminoids, their structure is used as the basis to design new drugs for the treatment of several types of cancers and microbial infections [1,7]. 
Curcuminoids are typically monitored at $420 \mathrm{~nm}$ based on their intense yellow color. The quantification of curcumin and related molecules in food samples entails an efficient separation of components to avoid mutual interferences. Two recent critical reviews can be found elsewhere $[8,9]$. Liquid chromatography with spectrometric detection seems to be a good choice for the determination of curcumin and other principal curcuminoids [10,11]. As pointed out by Ali et al. [12], reversed-phase HPLC using C18 columns is commonly used although anion exchange and micellar chromatography have also been introduced. In addition, the most volatile fraction of components can be better separated by gas chromatography [13]. Enhanced selectivity and sensitivity can be gained from mass spectrometry (MS) detection [14]. The characterization of curcuminoid structures have been based on LC-MS and LC-tandem MS [15,16], sometimes, combined with nuclear magnetic resonance (NMR) spectroscopy $[17,18]$. Apart from the range of applications to food analysis, some recent publications related to bioanalysis concern the characterization of curcumin metabolites from biological matrices by LC-MS [19,20]. Alternative separation methods take advantage of the great analytical performance of capillary electrophoresis (CE) under various electrophoretic modes [21-24]. Simpler methods based on non-chromatographic techniques entailing spectroscopic measurements by UV-Vis spectroscopy [25], fluorescence [26], FTIR spectroscopy [27] and electrochemical devices [28-30].

Apart from curcuminoids, another important group of turmeric components consists of phenolic acids and flavonoids, which exhibits a great descriptive ability for characterization, classification and authentication studies [31,32]. In this regard, profiling methods for the simultaneous determinations of several analytes rely on chromatographic techniques. Complementary estimations of overall indexes of antioxidant power have been carried out using various spectroscopic methods [33,34].

Strategies for the characterization, classification and authentication of turmeric samples typically rely on chemometric methods for a more comprehensive assessment of relevant descriptors, patterns, and relationships. Some researchers have recently explored the possibilities of curcuminoids as a source of analytical information using concentrations of some curcuminoids to discriminate among turmeric products using principal component analysis (PCA) [35]. Ni and coworkers exploited chromatographic fingerprints of extracts from various Chinese provinces by PCA and K-nearest neighbor (KNN) [36]. Kulyal and coworkers applied PCA to the characterization of turmeric cultivars, concluding that curcuminoid levels could be used to assess the turmeric quality and discriminate among the plant varieties [37]. Apart from chromatographic data, NMR fingerprints were also applied to authentication studies [38]. Combined information from different sources such as UV, FTIR, ${ }^{1} \mathrm{H}-\mathrm{NMR}$ in addition to HPLC was applied to obtain metabolic fingerprints to assess turmeric quality by PCA and hierarchical cluster analysis (HCA) [39].

This work deals with a HPLC-UV-Vis fingerprint approach to generate proper descriptors to be used in turmeric classification and discrimination. Customized extractions procedures were developed depending on the family of compounds of interest based on design of experiments (DoE). In this regard, methanol $(\mathrm{MeOH})$ was recommendable for an efficient recovery of curcuminoids while dimethyl sulfoxide (DMSO) was more appropriate for phenolic components. Besides, specific separation and detection conditions were established in the two cases using DoE as a very efficient way to get a great separation performance with fast chromatographic analysis. Data consisting of chromatographic fingerprints of both curcuminoid and phenolic systems from the analysis of commercial samples was further treated by chemometric methods to tackle the characterization of turmeric and curry samples. Interesting conclusions on sample characteristics and spice varieties could be drawn from the interpretation of principal component analysis (PCA) results. 


\section{Materials and Methods}

\subsection{Chemicals and Solutions}

Mobile phases for the chromatographic methods were prepared with formic acid $(>96 \%$, Sigma-Aldrich, St. Louis, MO, USA), acetonitrile (UHPLC-Supergradient, Panreac, Barcelona, Spain), Methanol (UHPLC-Supergradient, Panreac) and purified water with an Elix 3 Milli-Q purification system (Millipore, Bedford, MA, USA). Dimethyl sulfoxide (DMSO, $>99 \%$ v/v, Merck, Hohenbrunn, Bayern, Germany) and methanol were used as the extraction solvents.

Pure stock solutions of curcumin (cur, 98\%, Sigma-Aldrich, Steinheim, Germany), demethoxycurcumin (dmc, $>95 \%$, Biopurify Chemicals Ltd., Chengdu, Sichuan, China) and bisdemethoxycurcumin (bdmc, $>95 \%$, Biopurify Chemicals Ltd.) were prepared at a concentration of $1 \mathrm{~g} \mathrm{~L}^{-1}$ in methanol. The composition of polyphenolic profiles was checked with the following compounds: Arbutin $(>98 \%)$, caffeic acid $(>99 \%)$, caftaric acid $(>97 \%),(+)$-catechin $(>98 \%)$, chlorogenic acid (>95\%), p-coumaric acid (>99\%), (-)-epicatechin ( $>99), 3,4$-dihydroxybenzaldehid ( $>97 \%)$, ferulic acid $(>99 \%)$, gallic acid $(>99 \%)$, gentisic acid $(>98 \%)$, homogentisic acid $(>99 \%)$, homovanillic acid $(>99 \%)$, kaempferol $(>97 \%)$, morin $(>97 \%)$, myricetin $(>96 \%)$, polydatin $(>95 \%)$, protocatechuic acid $(>97 \%)$, quercetin $(>97 \%)$, resveratrol $(>99 \%)$, rutin $(>94 \%)$, sinapic acid $(>98 \%)$, syringic acid $(>97 \%)$, vanillic acid $(>97 \%)$, all of them from Sigma-Aldrich. Working standard solutions for HPLC for identification and quantification purposes were in the range 20 to $0.01 \mathrm{mg} \mathrm{L}^{-1}$ in $\mathrm{MeOH}$ :water $(1: 1, v: v)$.

\subsection{Samples and Sample Treatment}

Turmeric and curry samples were purchased from different retail stores of Barcelona (Table 1). All the samples were collected in 2018 with the exception of one aged (Erode var. collected in 2012). Samples (ca. $10 \mathrm{mg}$ accurately weighed) were subjected to ultrasound-assisted extraction using a Branson 5510 bath (Branson Ultrasonics, Danbury, CT, USA) with $5 \mathrm{~mL}$ of proper solvent for $40 \mathrm{~min}$ at room temperature. In particular, $\mathrm{MeOH}$ and $\mathrm{DMSO}$ were used to recover curcuminoids and polyphenols, respectively. After sonication, samples were centrifuged at $3500 \times g$ using a Rotanta centrifuge 460 RS (Hettich, Tuttlingen, Germany) and filtered through Nylon membranes of $0.45 \mu \mathrm{m}$ pore size. Sample matrix remained undissolved as a solid residue was rejected. The resulting extracts were stored in dark vials at $4{ }^{\circ} \mathrm{C}$ until chromatographic analysis. Quality controls (QCs) were prepared by mixing equal volumes $(100 \mu \mathrm{L})$ of all sample extracts.

\subsection{Chromatographic Analysis}

The chromatographic system consisted of an Agilent Series 1100 HPLC Chromatograph (Agilent Technologies, Palo Alto, CA, USA) with a quaternary pump (G1311A), a degasser (G1322A), an automatic injection system (G1392A) and a diode array detector (G1315B). The Agilent ChemStation software was used for instrument control and data processing.

Method A: Polyphenolic fingerprints mainly associated to phenolic acids and flavanols were recorded at $280 \mathrm{~nm}$, using a Kinetex column (Phenomenex, Torrance, CA, $100 \times 4.6 \mathrm{~mm}$ I.D. with $2.6 \mu \mathrm{m}$ particle size). The components of the mobile phase were $0.1 \%(v / v)$ formic acid aqueous solution and methanol (MeOH). Compounds were eluted with a linear gradient from 5\% to 95\% $\mathrm{MeOH}$ in $20 \mathrm{~min}$. The column was cleaned for $2 \mathrm{~min}$ at $95 \% \mathrm{~B}$ and conditioned for $3 \mathrm{~min}$ at $5 \% \mathrm{MeOH}$. The injection volume was $5 \mu \mathrm{L}$ and the flow rate was $0.4 \mathrm{~mL} \mathrm{~min}^{-1}$.

Method B: For the generation of curcuminoid fingerprints at $420 \mathrm{~nm}$, the separation was carried out in a reversed-phase column X Terra MS C18 $(2.1 \times 100 \mathrm{~mm}, 3.5 \mu \mathrm{m}$ particle size, Milford, MA, EUA) equipped with a C18 Security Guard pre-column $(4.0 \times 3.0 \mathrm{~mm}$ I.D.). The components of the mobile phase were $0.1 \%(v / v)$ formic acid aqueous solution and acetonitrile $(\mathrm{ACN})$. The elution gradient consisted of a linear increase from $40 \%$ to $90 \% \mathrm{ACN}$ in $20 \mathrm{~min}$. Subsequently, the column was cleaned for $2 \mathrm{~min}$ at $90 \% \mathrm{ACN}$ and conditioned for $5 \mathrm{~min}$ at $40 \% \mathrm{~B}$. The injection volume was $10 \mu \mathrm{L}$ and the flow rate was $0.4 \mathrm{~mL} \mathrm{~min}^{-1}$. 
Table 1. List of samples analyzed and their characteristics.

\begin{tabular}{|c|c|c|c|}
\hline Sample Type & Brand & Number of Samples * & Composition \\
\hline \multirow{16}{*}{ Turmeric } & Hacendado & 5 & curcuma longa (Erode) \\
\hline & MG & 1 & curcuma longa (Alleppey) \\
\hline & Burriac & 1 & curcuma longa \\
\hline & Carmencita & 1 & curcuma longa (Erode) \\
\hline & Ducros & 1 & curcuma longa \\
\hline & Artemis Bio & 1 & curcuma longa \\
\hline & Natco & 1 & curcuma longa \\
\hline & Pelotari & 1 & Unknown \\
\hline & Dani & 2 & curcuma zedoaria \\
\hline & Especies & 1 & curcuma longa (Alleppey) \\
\hline & Onena & 1 & curcuma longa (Madras) \\
\hline & Tata Sampann & 1 & Unknown \\
\hline & Herbalist & 1 & curcuma longa (Madras) \\
\hline & Street market & 1 & curcuma longa (Madras) \\
\hline & Biospirit & 1 & curcuma longa \\
\hline & NAAI & 1 & Unknown \\
\hline \multirow{8}{*}{ Curry } & Hacendado & 2 & $\begin{array}{l}\text { white pepper, coriander, ginger, cardamom, } \\
\text { clove, cinnamon, anise, mustard }\end{array}$ \\
\hline & Carrefour & 1 & $\begin{array}{l}\text { pepper, coriander, ginger, cumin, fenugreek, } \\
\text { laurel, fennel, mustard }\end{array}$ \\
\hline & Species Kania & 1 & $\begin{array}{l}\text { pepper, coriander, cumin, fenugreek, } \\
\text { parsley, chili, garlic, fennel }\end{array}$ \\
\hline & Condis & 1 & $\begin{array}{l}\text { pepper, coriander, fennel, cumin, cayenne, } \\
\text { garlic, anise }\end{array}$ \\
\hline & Burriac & 1 & $\begin{array}{l}\text { white pepper, coriander, ginger, cardamom, } \\
\text { clove, cinnamon, anise, mace }\end{array}$ \\
\hline & Eroski & 1 & $\begin{array}{c}\text { coriander, cardamom, ginger, fenugreek, } \\
\text { anise, garlic, clove, mustard }\end{array}$ \\
\hline & Ducros & 1 & $\begin{array}{l}\text { pepper, coriander, cumin, ginger, laurel, } \\
\text { anise, garlic, clove, cinnamon, mace }\end{array}$ \\
\hline & Street market & 1 & Unknown \\
\hline
\end{tabular}

${ }^{*}$ Number of containers collected in different locations.

\subsection{Data Analysis}

Exploratory and classification studies were carried out using the PLS-Toolbox (Eigenvector Research, Manson, WA, USA) working with MATLAB [40]. Detailed information on the theoretical background of the chemometric methods is given elsewhere [41].

Chromatographic fingerprints of curcuminoids and phenolic compounds were arranged in data matrices with rows representing samples (30 samples, see Table 1) and columns variables (time). In particular, for polyphenolics, data consisted of absorbance values taken at $280 \mathrm{~nm}$ in step of $0.0064 \mathrm{~min}$ within the working range 16.26 to $22.90 \mathrm{~min}$, thus the dimension of the corresponding data matrix was $30 \times 1035$. In the case of curcuminoids, absorbance values were taken at $420 \mathrm{~nm}$ from 6 to $22 \mathrm{~min}$ so that the dimension of the data matrix was $30 \times 2493$.

In order to improve the quality of the model, a precision filter based on the QC data reproducibility was applied on the chromatographic domain so that variables with high variability (RSD values higher than 20\%) were discarded. Data was normalized to provide similar weights for all the samples. For exploratory purposes, the plots of scores showing the distribution of the samples on the principal components (PCs) were used to investigate patterns concerning sample characteristics, such as variety or origin. Besides, the location of QCs in this graph was used to evaluate the robustness of the model. The plot of loadings showed the distribution of variables and allowed to infer their influence on the sample properties. In this way, the most relevant variables from the descriptive point of view could be detected. 


\section{Results and Discussion}

This work deals with a HPLC-UV-Vis fingerprint approach to generate proper descriptors to be used in turmeric classification and discrimination. In fingerprinting, the characterization relies on complex instrumental signals coming from known and unknown components present in the samples. It should be noted that quantitative information is implicitly embedded in the data since, reasonably, higher or lower peaks will be obtained from higher or lower compound concentrations. In this context, the quantification, identification or even knowledge of those components responsible for the instrumental data is not necessary. As a result, complex and time-consuming analytical steps devoted to quantitative determinations can be omitted without losing discrimination or authentication ability. Under this point of view, the extraction and chromatographic performances were evaluated in terms of number of extracted components (defined as the number of resolved peaks in the compositional fingerprints) rather than their complete recovery or quantitative determination.

\subsection{Optimization of the Extraction}

The recovery of polyphenolic and curcuminoid species from turmeric and curry samples was preliminarily assessed by rotary shaking, maceration and ultrasound-assisted extraction (UAE), using the overall chromatographic area as the analytical parameter to account the extraction rate. Studies were planned by DoE at two levels of solvent volume ( 5 and $10 \mathrm{~mL}$ ), using 90 min extraction time and $5 \mathrm{mg}$ of a representative turmeric sample. The extraction technique strongly affected the extraction performance, being UAE much more efficient so it was selected for further experiments. Conversely, within the experimental domain under study, the influence of solvent volume was not relevant. The effect of processing time in UAE was studied in the range 5 to $90 \mathrm{~min}$. It was found that the response increased with time from 5 to $30 \mathrm{~min}$ and remained constant from 30 to $90 \mathrm{~min}$. Then, an extraction time of $40 \mathrm{~min}$ was chosen. The effect of the temperature of the ultrasound bath was assayed at room temperature and $40{ }^{\circ} \mathrm{C}$. Peak areas were similar in the two cases so, for practical reasons, further studies were carried out at room temperature.

Complementary experiments were designed to evaluate thoroughly the influence of the solvent on the compositional fingerprints of both polyphenols and curcuminoids. The main goal of this research was obtaining the richest profiles that could contain a wide variety of features (chromatographic peaks of known or unknown species) with putative descriptive or discriminant capability. Thus, the criterion for the selection of the extraction solvent relied on providing the greatest number of peaks at the working wavelengths. Various solvents with different polarities were chosen in this study, including methylene chloride $\left(\mathrm{CH}_{2} \mathrm{Cl}_{2}\right), \mathrm{ACN}$, ethanol $(\mathrm{EtOH}), \mathrm{MeOH}, \mathrm{DMSO}$ and mixtures of $\mathrm{MeOH} /$ water $(1 / 1, v / v)$ and DMSO/water $(1 / 1, v / v)$.

Regarding polyphenolic profiles, extracts were analyzed chromatographically using the Kinetex column. The elution gradient applied consisted of increasing the percentage of methanol from $5 \%$ to $95 \%$ in $15 \mathrm{~min}$, a further cleaning step at $95 \%$ methanol for $2 \mathrm{~min}$ and returning to the initial conditions. Information gained from the injection of polyphenol standards showed that, under these conditions, phenolic acid eluted within the time window 2 to $7 \mathrm{~min}$ and most of flavonoids from 7 to $10 \mathrm{~min}$ (please note that these studies were carried out with a preliminary chromatographic method, not the optimum one). As shown in Figure 1, the composition of the lixiviates strongly depended on the composition of the solvent. ACN and DMSO resulted in the most interesting cases for discussion as they provided the richest chromatograms. The comparison with standards revealed that ACN extracts were poorer in polyphenolic components while they showed quite intense peaks for low polar compounds. This fraction might be related to sesquiterpenoids such as turmerones and others. Conversely, DMSO extracts contained abundant polyphenolic species as deduced from the corresponding standards. Although both solvents displayed complementary performance, DMSO was finally selected because of the higher complexity of the resulting polyphenolic fingerprints (components mainly extracted with ACN are currently outside the scope of this paper). 


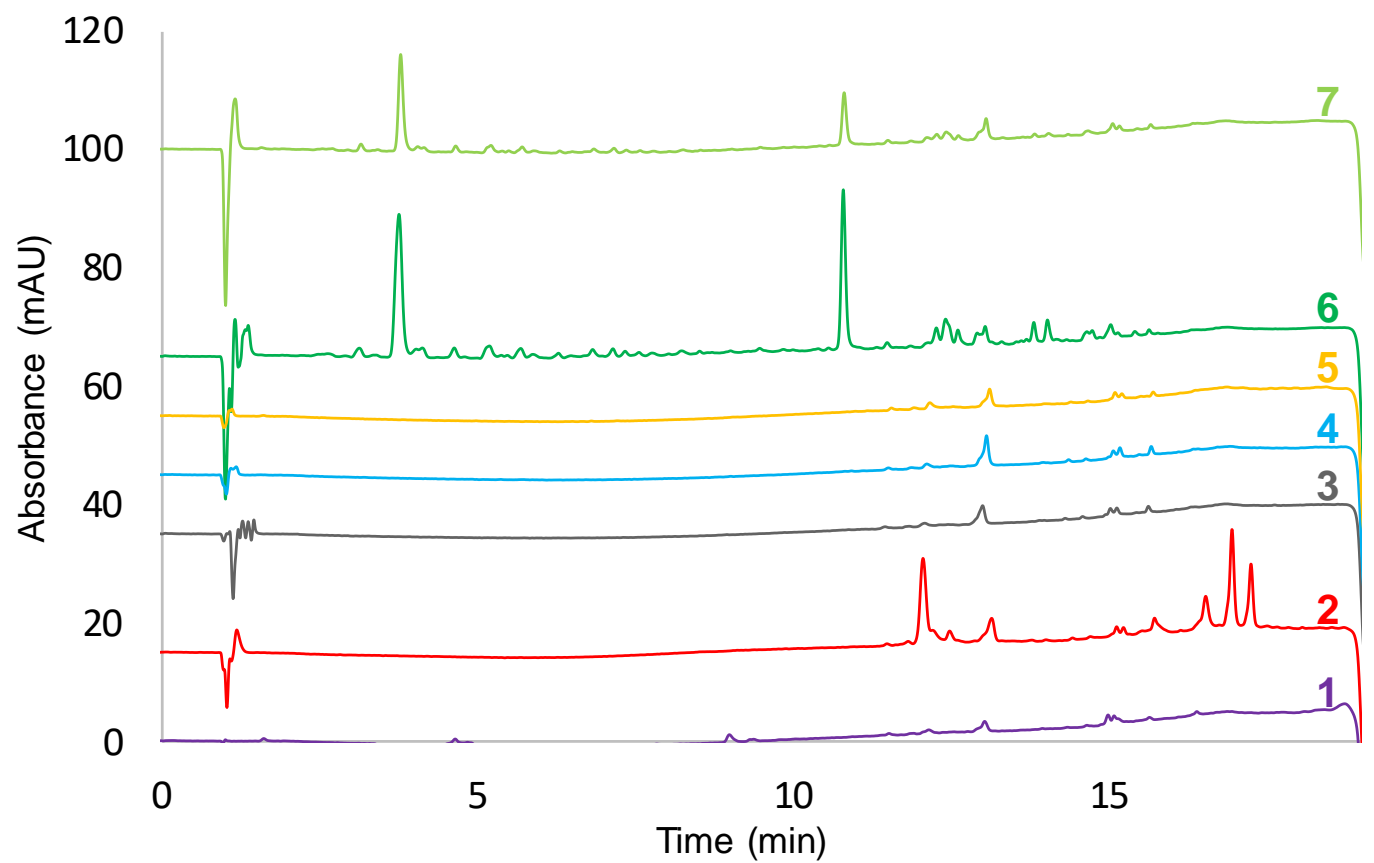

Figure 1. Chromatograms recorded at $280 \mathrm{~nm}$ (method A) corresponding to the extraction of a turmeric sample (Curcuma longa, Erode var.). Extraction conditions: A total of $5 \mathrm{mg}$ of sample; $10 \mathrm{~mL}$ solvent; 40 min extraction time with ultrasound-assisted extraction (UAE). Separation conditions: Kinetex column; elution gradient $5 \%$ to $90 \% \mathrm{MeOH}$ in $15 \mathrm{~min}$. Solvent assignment: $1=\mathrm{CH}_{2} \mathrm{Cl}_{2} ; 2=$ acetonitrile $(\mathrm{ACN}) ; 3=\mathrm{EtOH} ; 4=\mathrm{MeOH} ; 5=\mathrm{MeOH} /$ water $(1 / 1, v / v) ; 6=\mathrm{DMSO} ; 7=\mathrm{DMSO} /$ water $(1 / 1, v / v)$.

In the case of curcuminoid fingerprints, extracts were analyzed at $420 \mathrm{~nm}$ using the $X$ Terra column with an elution gradient based on the increase of the ACN percentage from $40 \%$ to $90 \%$ in $20 \mathrm{~min}$ and return to the initial conditions. The number and distribution of the species were similar in all the cases, regardless of the solvent used. Chromatograms contained various peaks of species with absorption maxima at ca. $420 \mathrm{~nm}$, including cur, $\mathrm{dmc}$ and bdmc and other unknown species (see Figure 2). Contents of cur, dmc and bdmc in the samples are given elsewhere [35]. For the rest of compounds, their full identification is still pending. Anyway, LC-MS experiments showed that compounds labelled as 2, 3 and 4 were tentatively assigned as cyclocurcumin, cyclodemethoxycurcumin and cyclobisdemethoxycurcumin as their $\mathrm{m} / \mathrm{z}$ values exactly matched those of cur, dmc and bdmc, respectively (to be confirmed since standards were not available). Compound labelled 10 also corresponded to another (unknown) curcuminoid, slightly more apolar, while 9 and 8 were its demethylated and bisdemethylated counterparts, exhibiting $m / z$ differences of -14 and -28 , respectively. Anyway, more intense peaks were obtained with $\mathrm{MeOH}$ so that this solvent was recommendable for the extraction of curcumin-related species [35]. 


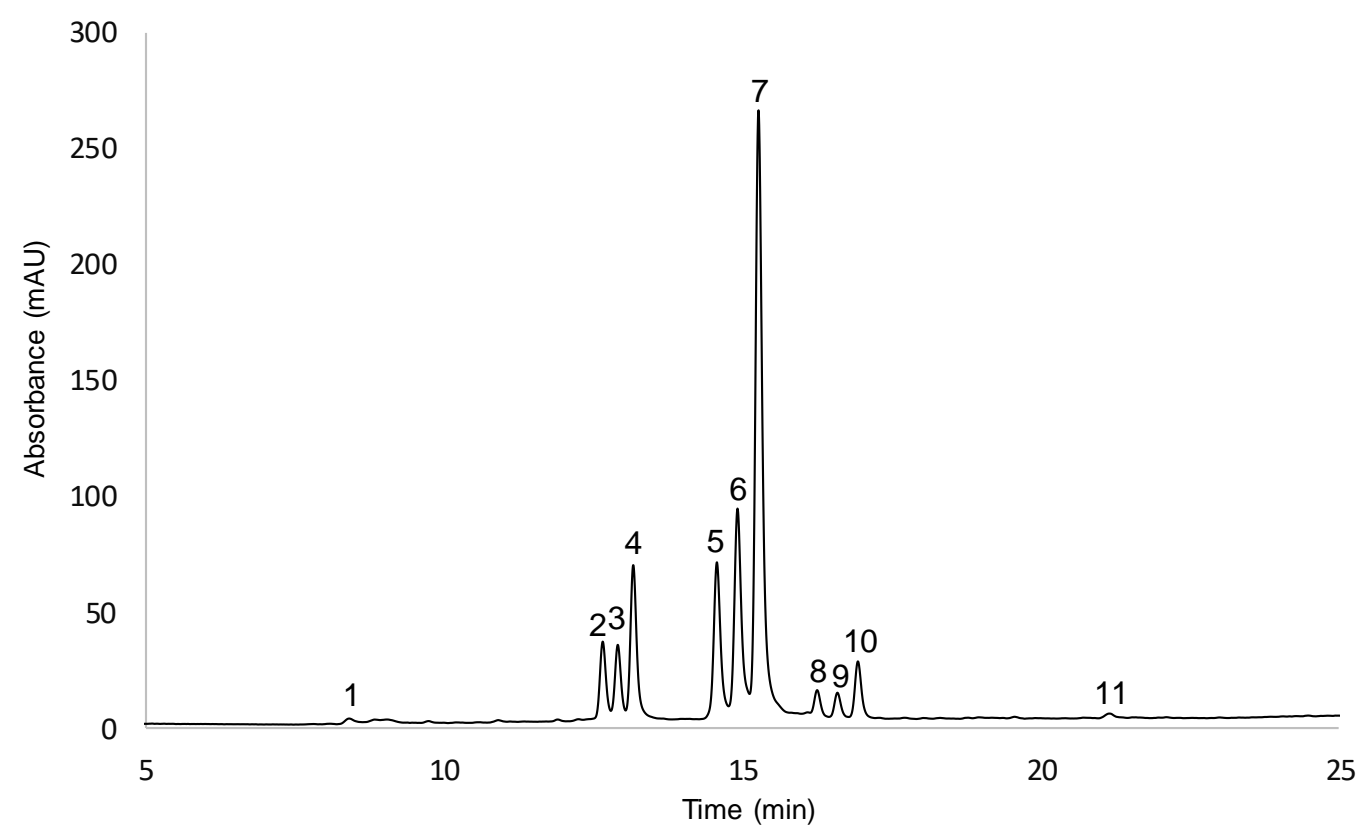

Figure 2. Chromatograms recorded at $420 \mathrm{~nm}$ (method B) corresponding to the extraction of a turmeric sample (Curcuma longa, Erode var.) with methanol. Extraction conditions: A total of $5 \mathrm{mg}$ of sample; $10 \mathrm{~mL} \mathrm{MeOH}$; 40 min extraction time with UAE. Separation conditions: X Terra column; elution gradient $40 \%$ to $90 \%$ ACN in 20 min. Peak assignment: $5=$ bisdemethoxycurcumin; $6=$ demethoxycurcumin; 7 = curcumin; 1 to 4 and 8 to 11 were other (unknown) curcuminoids.

\subsection{Optimization of the Separation Conditions}

Owing to the remarkable differences in the physicochemical features of curcuminoids and phenolic acids, specific chromatographic methods should be applied to generate optimal fingerprints for the two families of descriptors. Regarding polyphenolic species, the chromatographic method was adapted from some recent publications that proved the great performance of reversed-phase core-shell Kinetex columns to separate phenolic acids and flavonoids [42,43]. Depending on the sample, customized elution gradients were designed using $0.1 \%(v / v)$ formic acid aqueous solution and methanol as the components of the mobile phase, and working at a flow rate of $0.4 \mathrm{~mL} \mathrm{~min}^{-1}$. Unfortunately, this option provided a poor performance in the case of curcuminoids and components such as cur, dmc and bdmc could not be mutually resolved. Preliminary studies suggested that the X Terra MS C18 column working with $0.1 \%(v / v)$ formic acid aqueous solution and $\mathrm{ACN}$ as the solvents was highly recommendable for separation on curcuminoids. In this case, the flow rate should be limited to $0.4 \mathrm{~mL} \mathrm{~min}^{-1}$ to keep the maximum pressure below 360 bar. The suitability of the X Terra MS C18 column for the separation of polyphenols was also investigated. Results indicated that, under the best conditions, the number of phenolic acids and flavonoids resolved was lower than that using the Kinetex counterpart. Hence, their simultaneous integration in a single-run method was considered inviable because important information might be lost.

The separation of the polyphenolic components of turmeric and related samples was based on DoE to get a better resolution of putative descriptors of interest in sample discrimination and classification. Again, solvent percentage and gradient time were the factors under evaluation, and the number of resolved peaks and analysis time the objectives of the optimization. Starting $\mathrm{MeOH}$ percentages to be assayed were $5 \%, 10 \%$ and $15 \%$ and gradient times 20,30 and $40 \mathrm{~min}$. The optimal separation was reached under a desirability function accounting as a compromise among resolved peaks and retention time $\left(t_{R}\right)$ of the most retained compound, according to the following expression: $D=\left(d_{\text {peaks }} \times d t_{R}\right)^{1 / 2}$, being $d_{\text {peaks }}$ and $d t_{R}$ the desirability contributions of the number of peaks and time, respectively. The relationship among peaks and $d_{\text {peaks }}$ was accounted under the assumption that less than 20 peaks 
was unacceptable $(d=0)$ and more that 50 peaks was fully satisfactory $(d=1)$. In the case of $t_{R}$, more than 35 min were unacceptable $(d=0)$ and less than 15 min was fully satisfactory $(d=1)$. As a result, the best conditions corresponded to a gradient profile increasing the $\mathrm{MeOH}$ percentage from $5 \%$ to 95\% in 20 min (Figure 3a).

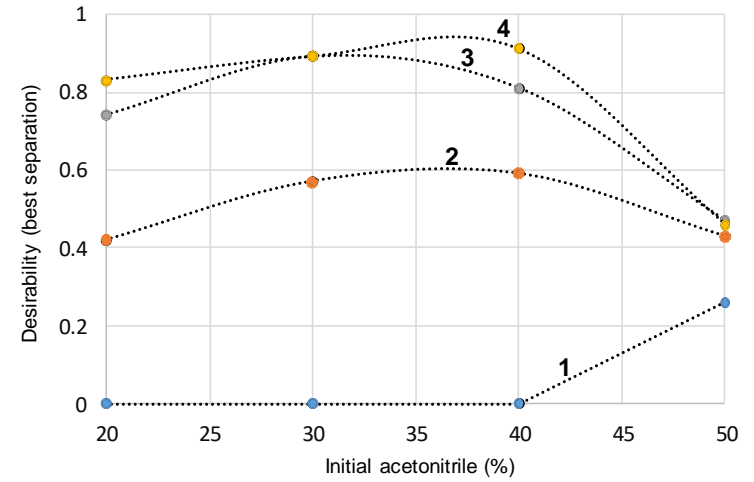

(b)

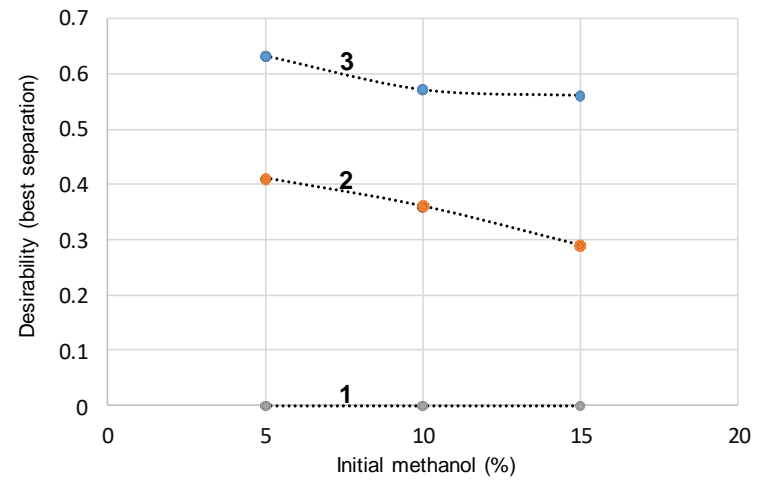

(a)

Figure 3. Optimization of the separation of phenolic compounds (method A) and curcuminoids (method B) by experimental design. (a) Phenolic compounds. Conditions of gradient time: $1=30 \mathrm{~min}$; $2=40 \mathrm{~min} ; 3=50 \mathrm{~min}$. (b) Curcuminoids. Conditions of gradient time: $1=5 \mathrm{~min} ; 2=10 \mathrm{~min}$; $3=15 \mathrm{~min} ; 4=20 \mathrm{~min}$.

For curcumin-related species, the moderate hydrophobicity of these molecules, with logP values around 3.5, required the use of intermediate or high percentages of organic solvents for their elution under reversed-phase mode. This is the most common choice among the recent publications on this topic as pointed out elsewhere $[10,11]$. The use of phenyl-based columns can even improve the interaction of the analytes to achieve better separations [12]. Besides, it was observed that $\mathrm{MeOH}$ provided poor selectivity to resolve cur, $\mathrm{dmc}$ and bdmc peaks while ACN led to excellent separations [35]. Finer optimization of the elution gradient was carried out by DoE considering initial ACN percentage $(20 \%, 30 \%, 40 \%$ and $50 \%)$ and gradient time $(5,10,15$ and $20 \mathrm{~min})$ as the target factors. In any case, final ACN\% was 90 and further column washing ( $2 \mathrm{~min}$ at $90 \%$ ) and column conditioning (5 min at the initial ACN\%) were applied. Selected working conditions providing full resolution of cur, $\mathrm{dmc}$ and bdmc in the minimum analysis time corresponded to a gradient profile from $40 \%$ to $90 \%$ ACN in 20 min (Figure 3b). Under these circumstances, repeatabilities for the retention time of the peaks of cur, dmc and bdmc were below $0.4 \%$, and for the peak areas were below $2 \%$.

\subsection{Sample Characterization by PCA}

The scientific literature describes various publications for the characterization and authentication of turmeric products based on compositional fingerprints and chemometrics. The idea of UV-Vis chromatograms as the source of analytical information has been scarcely exploited [36] and, in general, authors rely on spectroscopic techniques exhibiting high detection performance such as FTIR, NMR or MS $[27,38,39]$. In this section, the latent information occurring in the polyphenolic and curcuminoid HPLC UV-Vis fingerprints will be analyzed chemometrically to try to find out patterns of interest for description and authentication purposes.

Regarding polyphenolic fingerprinting, data under study consisted of sample chromatograms at $280 \mathrm{~nm}$ taken in the working range 16.26 to $22.9 \mathrm{~min}$, in which the most significant components were eluted, especially those corresponding to hydroxybenzoic and hydroxycinnamic acids and their derivatives, and various flavonoids. Peaks of some abundant compounds were found to be important descriptors to explain the distribution of turmeric samples as a function of varietal classes. PCA was able to retain a more than $78 \%$ of data variance with two components $(71.1 \%$ from PC1 and $7.6 \%$ from PC2). The map of scores (Figure 4) clearly separated pure turmeric from other preparations containing 
additional species such as pepper, coriander, ginger, cardamom, clove, cinnamon, anise, mustard, parsley, garlic, etc. Hence, turmeric samples were mainly distributed on the right side of the plot while mixtures with other spices were located to the left side. Besides, QCs appeared in a compact group in the center of the model, thus indicating the great reproducibility of the chromatographic runs as well as the robustness of the statistical analysis. Regarding varietal origin, Curcuma longa (Erode variety) were mainly found on the bottom-right sector, while other species and varieties spread out on the top-right area.

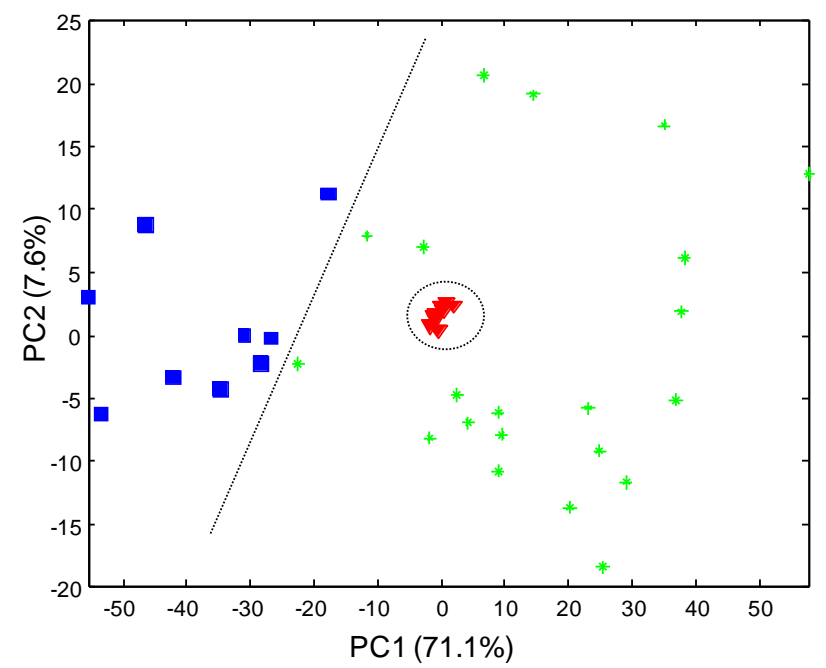

Figure 4. Map of scores of PC1 versus PC2 of turmeric and curry samples based on polyphenolic fingerprints at $280 \mathrm{~nm}$. Conditions: Chromatographic method A, working time window 16.26 to $22.90 \mathrm{~min}$, data dimension $30 \times 1035$. Sample assignment: Star $=$ turmeric sample; square $=$ curry sample; triangle $=$ quality control.

For a more detailed discrimination among the turmeric varieties, the capability of polyphenolic profiles was found to be limited so the next models were focused on curcuminoids as potential sample descriptors. In this regard, chromatograms at $420 \mathrm{~nm}$ in the working window containing the major curcuminoid components (from 6 to $22 \mathrm{~min}$ ) were used as the data. The PCA model captured almost a $70 \%$ of variance with 2 PCs (55.8\% from PC1 and 13.3\% from PC2). The plot of scores (Figure 5) showed good discrimination between varieties, in which Erode and Alleppey samples were located on the bottom- and top-right areas and Madras samples were found on the left side. This approach was also able to detect expired samples, which were located far away from the regular ones (see sample labeled as $\mathrm{E}^{*}$ ). In connection with the study of loadings, it was deduced that Erode samples were richer in curcuminoids, especially cur, dmc and bdmc. Alleppey products showed a higher proportion of $\mathrm{dmc}$ and bdmc than cur, and this characteristic trend could be exploited for the identification. Finally, the Madras variety displayed high peaks of various more polar (still unknown) curcuminoids. 


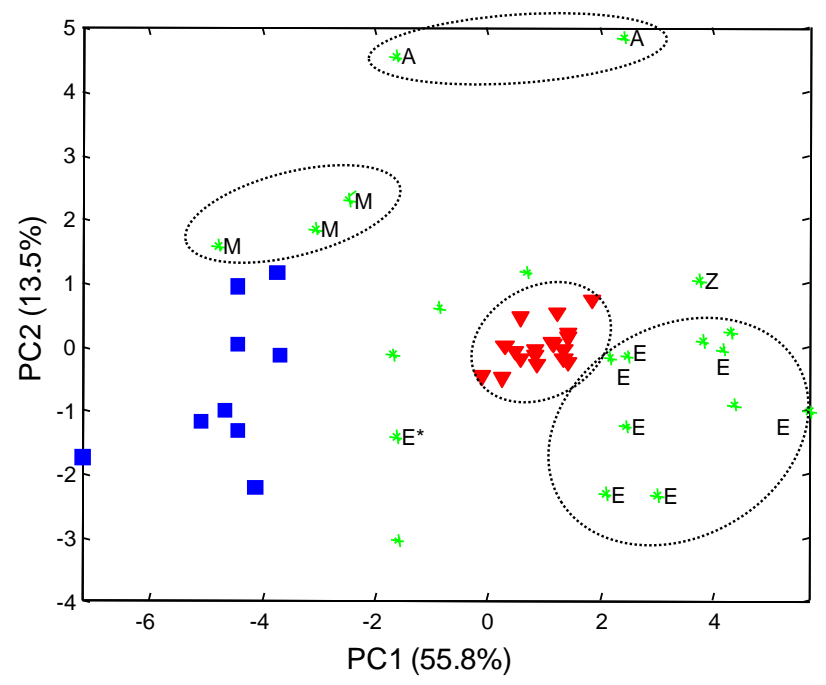

Figure 5. Map of scores of PC1 versus PC2 of turmeric and curry samples based on curcuminoid fingerprints at $420 \mathrm{~nm}$. Conditions: Chromatographic method B, working time window 6 to $22 \mathrm{~min}$, data dimension $30 \times 2493$. Sample assignment: Star $=$ turmeric sample; square $=$ curry sample; triangle $=$ quality control. Variety assignment: $\mathrm{E}=$ Erode; $\mathrm{M}=$ Madras; $\mathrm{A}=$ Allepey; $\mathrm{Z}=$ Zeodaria.

\section{Conclusions}

Two different procedures were considered in this work to provide information dealing with curcuminoids and polyphenolic species to be used as potential markers of turmeric features. Indeed, the use of a single method for dealing with the two families of components simultaneously may provide some practical advantages at the expense of having less detailed fingerprints. Hence, it was guessed that some important descriptors may be lost. Optimal extraction and separation conditions, successfully established by experimental design, differed depending on the target analytes. More specifically, dimethyl sulfoxide and methanol were recommended to recover phenolic and curcumin-related molecules, respectively. In terms of separation performance, best separation conditions leading to the richest phenolic and curcuminoid profiles were obtained using different methods, involving different analytical columns and elution gradient programs. Data resulting from the analysis of a set of turmeric and curry samples according to these two options (e.g., fingerprints at $280 \mathrm{~nm}$ for phenolic compounds and fingerprints at $420 \mathrm{~nm}$ for curcuminoids) were treated chemometrically to obtain an overall picture of the behavior of samples and the identification of descriptive variables. In this regard, principal component analysis revealed distinctive patterns about the plant varieties, thus resulting in a good option to deal with classification and authentication issues. In particular, polyphenolic fingerprints discriminated among turmeric and other food flavoring mixtures while curcuminoid counterparts contributed to separate classes of turmeric varieties.

The descriptive capacity of chromatograms from ACN extracts has been not investigated yet but, in the future, this option may open up new analytical possibilities. Other studies are in progress to try to identify the structures of unknown compounds by LC-MS (/MS) and other instrumental techniques.

Author Contributions: Conceptualization, O.N. and J.S.; methodology, O.V.-C. and S.S.; investigation, O.V.-C. and N.N.; writing - original draft preparation, O.V.-C. and J.S.; writing—review and editing, O.N., S.S. and J.S. All authors have read and agreed to the published version of the manuscript.

Funding: This research was supported by the Agency for Administration of University and Research Grants (Generalitat de Catalunya, Spain) under the projects 2017SGR-171 and 2017SGR-310.

Conflicts of Interest: The authors declare no conflicts of interest. 


\section{References}

1. Kotha, R.R.; Luthria, D.L. Curcumin: Biological, Pharmaceutical, Nutraceutical, and Analytical Aspects. Molecules 2019, 24, 2930. [CrossRef] [PubMed]

2. Sasikumar, B. Genetic Resources of Curcuma: Diversity, Characterization and Utilization. Plant Genet. Resour. 2005, 3, 230-251. [CrossRef]

3. Balasubramanian, S.; Roselin, P.; Singh, K.K.; Zachariah, J.; Saxena, S.N. Postharvest Processing and Benefits of Black Pepper, Coriander, Cinnamon, Fenugreek, and Turmeric Spices. Crit. Rev. Food Sci. Nutr. 2016, 56, 1585-1607. [CrossRef]

4. Nair, K.L.; Thulasidasan, A.K.T.; Deepa, G.; Anto, R.J.; Kumar, G.S.V. Purely Aqueous PLGA Nanoparticulate Formulations of Curcumin Exhibit Enhanced Anticancer Activity with Dependence on the Combination of the Carrier. Int. J. Pharm. 2012, 425, 44-52. [CrossRef]

5. Bhawana; Basniwal, R.K.; Buttar, H.S.; Jain, V.K.; Jain, N. Curcumin Nanoparticles: Preparation, Characterization, and Antimicrobial Study. J. Agric. Food Chem. 2011, 59, 2056-2061. [CrossRef]

6. Othman, R.; Abdurasid, M.A.; Mahmad, N.; Ahmad Fadzillah, N. Alkaline-Based Curcumin Extraction from Selected Zingiberaceae for Antimicrobial and Antioxidant Activities. Pigment Resin Technol. 2019, 48, 289-296. [CrossRef]

7. Da Silva, A.C.; de Santos, P.D.F.; do Silva, J.T.P.; Leimann, F.V.; Bracht, L.; Gonçalves, O.H. Impact of Curcumin Nanoformulation on Its Antimicrobial Activity. Trends Food Sci. Technol. 2018, 72, 74-82. [CrossRef]

8. Kotra, V.S.R.; Satyabanta, L.; Goswami, T.K. A Critical Review of Analytical Methods for Determination of Curcuminoids in Turmeric. J. Food Sci. Technol. 2019, 56, 5153-5166. [CrossRef]

9. Carolina Alves, R.; Perosa Fernandes, R.; Fonseca-Santos, B.; Damiani Victorelli, F.; Chorilli, M. A Critical Review of the Properties and Analytical Methods for the Determination of Curcumin in Biological and Pharmaceutical Matrices. Crit. Rev. Anal. Chem. 2019, 49, 138-149. [CrossRef]

10. Chao, I.C.; Wang, C.M.; Li, S.P.; Lin, L.G.; Ye, W.C.; Zhang, Q.W. Simultaneous Quantification of Three Curcuminoids and Three Volatile Components of Curcuma Longa Using Pressurized Liquid Extraction and High-Performance Liquid Chromatography. Molecules 2018, 23, 1568. [CrossRef] [PubMed]

11. Peram, M.R.; Jalalpure, S.S.; Joshi, S.A.; Palkar, M.B.; Diwan, P.V. Single Robust RP-HPLC Analytical Method for Quantification of Curcuminoids in Commercial Turmeric Products, Ayurvedic Medicines, and Nanovesicular Systems. J. Liq. Chromatogr. Relat. Technol. 2017, 40, 487-498. [CrossRef]

12. Ali, I.; Haque, A.; Saleem, K. Separation and identification of curcuminoids in turmeric powder by HPLC using phenyl column. Anal. Methods 2014, 6, 2526-2536. [CrossRef]

13. Hiserodt, R.; Hartman, T.G.; Ho, C.T.; Rosen, R.T. Characterization of Powdered Turmeric by Liquid Chromatography-Mass Spectrometry and Gas Chromatography-Mass Spectrometry. J. Chromatogr. A 1996, 740, 51-63. [CrossRef]

14. Ashraf, K.; Mujeeb, M.; Ahmad, A.; Ahmad, N.; Amir, M. Determination of Curcuminoids in Curcuma Longa Linn. by UPLC/Q-TOF-MS: An Application in Turmeric Cultivation. J. Chromatogr. Sci. 2015, 53, 1346-1352. [CrossRef] [PubMed]

15. Jiang, H.; Timmermann, B.N.; Gang, D.R. Characterization and Identification of Diarylheptanoids in Ginger (Zingiber Officinale Rosc.) Using High-Performance Liquid Chromatography/Electrospray Ionization Mass Spectrometry. Rapid Commun. Mass Spectrom. 2007, 21, 509-518. [CrossRef]

16. Jia, S.; Du, Z.; Song, C.; Jin, S.; Zhang, Y.; Feng, Y.; Xiong, C.; Jiang, H. Identification and Characterization of Curcuminoids in Turmeric Using Ultra-High Performance Liquid Chromatography-Quadrupole Time of Flight Tandem Mass Spectrometry. J. Chromatogr. A 2017, 1521, 110-122. [CrossRef]

17. Qiao, X.; Lin, X.H.; Ji, S.; Zhang, Z.X.; Bo, T.; Guo, D.A.; Ye, M. Global Profiling and Novel Structure Discovery Using Multiple Neutral Loss/Precursor Ion Scanning Combined with Substructure Recognition and Statistical Analysis (MNPSS): Characterization of Terpene-Conjugated Curcuminoids in Curcuma Longa as a Case Study. Anal. Chem. 2016, 88, 703-710. [CrossRef]

18. Jayaprakasha, G.K.; Nagana Gowda, G.A.; Marquez, S.; Patil, B.S. Rapid Separation and Quantitation of Curcuminoids Combining Pseudo Two-Dimensional Liquid Flash Chromatography and NMR Spectroscopy. J. Chromatogr. B Anal. Technol. Biomed. Life Sci. 2013, 937, 25-32. [CrossRef] 
19. Shi, M.; Gao, T.; Zhang, T.; Han, H. Characterization of Curcumin Metabolites in Rats by Ultra-High-Performance Liquid Chromatography with Electrospray Ionization Quadrupole Time-of-Flight Tandem Mass Spectrometry. Rapid Commun. Mass Spectrom. 2019, 33, 1114-1121. [CrossRef]

20. Jude, S.; Amalraj, A.; Kunnumakkara, A.B.; Divya, C.; Löffler, B.M.; Gopi, S. Development of Validated Methods and Quantification of Curcuminoids and Curcumin Metabolites and Their Pharmacokinetic Study of Oral Administration of Complete Natural Turmeric Formulation (Cureit ${ }^{\mathrm{TM}}$ ) in Human Plasma via UPLC/ESI-Q-TOF-MS Spectrometry. Molecules 2018, 23, 2415. [CrossRef]

21. Wu, C.; Wang, W.; Quan, F.; Chen, P.; Qian, J.; Zhou, L.; Pu, Q. Sensitive Analysis of Curcuminoids via Micellar Electrokinetic Chromatography with Laser-Induced Native Fluorescence Detection and Mixed Micelles-Induced Fluorescence Synergism. J. Chromatogr. A 2018, 1564, 207-213. [CrossRef] [PubMed]

22. Anubala, S.; Sekar, R.; Nagaiah, K. Determination of Curcuminoids and Their Degradation Products in Turmeric (Curcuma Longa) Rhizome Herbal Products by Non-Aqueous Capillary Electrophoresis with Photodiode Array Detection. Food Anal. Methods 2016, 9, 2567-2578. [CrossRef]

23. Kalaycioğlu, Z.; Hashemi, P.; Günaydin, K.; Erim, F.B. The Sensitive Capillary Electrophoretic-LIF Method for Simultaneous Determination of Curcuminoids in Turmeric by Enhancing Fluorescence Intensities of Molecules upon Inclusion into (2-Hydroxypropyl)- $\beta$-Cyclodextrin. Electrophoresis 2015, 36, 2516-2521. [CrossRef] [PubMed]

24. Li, F.; Liu, R.; Yang, F.; Xiao, W.; Chen, C.; Xia, Z. Determination of Three Curcuminoids in Curcuma Longa by Microemulsion Electrokinetic Chromatography with Protective Effects on the Analytes. Anal. Methods 2014, 6, 2566-2571. [CrossRef]

25. Unsal, Y.E.; Tuzen, M.; Soylak, M. Ultrasound-Assisted Ionic Liquid-Dispersive Liquid-Liquid of Curcumin in Food Samples Microextraction and Its Spectrophotometric Determination. J. AOAC Int. 2019, 102, $217-221$. [CrossRef]

26. Ali, Z.; Saleem, M.; Atta, B.M.; Khan, S.S.; Hammad, G. Determination of Curcuminoid Content in Turmeric Using Fluorescence Spectroscopy. Spectrochim. Acta Part A Mol. Biomol. Spectrosc. 2019, 213, 192-198. [CrossRef]

27. Lestari, H.P.; Martono, S.; Wulandari, R.; Rohman, A. Simultaneous Analysis of Curcumin and Demethoxycurcumin in Curcuma Xanthorriza Using FTIR Spectroscopy and Chemometrics. Int. Food Res. J. 2017, 24, 2097-2101.

28. Zokhtareh, R.; Rahimnejad, M. An Investigation of New Electrochemical Sensors for Curcumin Detection: A Mini Review. Anal. Methods 2019, 11, 4401-4409. [CrossRef]

29. Li, K.; Li, Y.; Yang, L.; Wang, L.; Ye, B. The Electrochemical Characterization of Curcumin and Its Selective Detection in Curcuma Using a Graphene-Modified Electrode. Anal. Methods 2014, 6, 7801-7808. [CrossRef]

30. Chaisiwamongkhol, K.; Ngamchuea, K.; Batchelor-Mcauley, C.; Compton, R.G. Multiwalled Carbon Nanotube Modified Electrodes for the Adsorptive Stripping Voltammetric Determination and Quantification of Curcumin in Turmeric. Electroanalysis 2017, 29, 1049-1055. [CrossRef]

31. Lucci, P.; Saurina, J.; Núñez, O. Trends in LC-MS and LC-HRMS Analysis and Characterization of Polyphenols in Food. TrAC Trends Anal. Chem. 2017, 88, 1-24. [CrossRef]

32. Saurina, J. Characterization of Wines Using Compositional Profiles and Chemometrics. TrAC Trends Anal. Chem. 2010, 29, 234-245. [CrossRef]

33. Sepahpour, S.; Selamat, J.; Manap, M.Y.A.; Khatib, A.; Razis, A.F.A. Comparative Analysis of Chemical Composition, Antioxidant Activity and Quantitative Characterization of Some Phenolic Compounds in Selected Herbs and Spices in Different Solvent Extraction Systems. Molecules 2018, 23, 402. [CrossRef] [PubMed]

34. Vallverdú-Queralt, A.; Regueiro, J.; Alvarenga, J.F.R.; Martinez-Huelamo, M.; Leal, L.N.; Lamuela-Raventos, R.M. Characterization of the Phenolic and Antioxidant Profiles of Selected Culinary Herbs and Spices: Caraway, Turmeric, Dill, Marjoram and Nutmeg. Food Sci. Technol. 2015, 35, 189-195. [CrossRef]

35. Vidal, O.; Castilla, X.; Aliaga-Alcalde, N.; López-Periago, A.; Domingo, C.; Sentellas, S.; Saurina, J. Determination of Curcuminoids by Liquid Chromatography with Diode Array Detection. Application to the Characterization of Turmeric and Curry Samples. Curr. Anal. Chem. 2020, 16, 95-105. [CrossRef]

36. Ni, Y.; Mei, M.; Kokot, S. Resolution of High Performance Liquid Chromatographic Fingerprints of Rhizoma Curcumae by Application of Chemometrics. J. Liq. Chromatogr. Relat. Technol. 2011, 34, 1952-1964. [CrossRef] 
37. Kulyal, P.; Kuchibhatla, L.N.; Uma Maheshwari, K.; Nirmal Babu, K.; Tetali, S.D.; Raghavendra, A.S. Highly Sensitive HPLC Method for Estimation of Total or Individual Curcuminoids in Curcuma Cultivars and Commercial Turmeric Powders. Curr. Sci. 2016, 111, 1816-1824. [CrossRef]

38. Windarsih, A.; Rohman, A.; Swasono, R.T. Application of 1H-NMR Based Metabolite Fingerprinting and Chemometrics for Authentication of Curcuma Longa Adulterated with C. Heyneana. J. Appl. Res. Med. Aromat. Plants 2019, 13, 100203. [CrossRef]

39. Gad, H.A.; Bouzabata, A. Application of Chemometrics in Quality Control of Turmeric (Curcuma Longa) Based on Ultra-Violet, Fourier Transform-Infrared and 1H NMR Spectroscopy. Food Chem. 2017, 237, 857-864. [CrossRef]

40. Wise, B.; Gallager, N.B. PLS_Toolbox for Use with MATLAB, Version 2.0; Eigenvector Research Inc.: Mason, WA, USA, 1992.

41. Massart, D.L.; Vandeginste, B.G.M.; Buydens, L.M.C.; Jong, S.; Lewi, P.J.; Smeyers-Verbeke, J. Handbook of Chemometrics and Qualimetrics; Elsevier: Amsterdam, The Netherlands, 1997.

42. Izquierdo-Llopart, A.; Saurina, J. Characterization of Sparkling Wines According to Polyphenolic Profiles Obtained by HPLC-UV/Vis and Principal Component Analysis. Foods 2019, 8, 22. [CrossRef]

43. Campmajo, G.; Navarro, G.J.; Nunez, N.; Puignou, L.; Saurina, J.; Nunez, O. Non-Targeted HPLC-UV Fingerprinting as Chemical Descriptors for the Classification and Authentication of Nuts by Multivariate Chemometric Methods. Sensors 2019, 19, 1388. [CrossRef] [PubMed]

(C) 2020 by the authors. Licensee MDPI, Basel, Switzerland. This article is an open access article distributed under the terms and conditions of the Creative Commons Attribution (CC BY) license (http://creativecommons.org/licenses/by/4.0/). 